\title{
Entanglement, Upper Probabilities and Decoherence in Quantum Mechanics
}

\author{
Stephan Hartmann* and Patrick Suppes ${ }^{\dagger}$ \\ *Center for Logic and Philosophy of Science, Tilburg University, 5000 LE Tilburg, The \\ Netherlands and `Stanford University, 220 Panama Street, Stanford, CA 94305-4101, USA
}

\begin{abstract}
Computation of decay time for entangled quantum systems is an important aspect of decoherence theories. Here we explore this topic from the standpoint of computing the decay time to the existence of a joint probability distribution of the entangled particles - atoms, in our case. We also analyze the problem from the viewpoint of the decay of an improper upper probability distribution, for the entangled particles and its continuous decay into a proper probability distribution. A standard quantum decoherence model and the upper-probability model have, it turns out, the same expected decay time for a familiar example of a system with a Bell state.
\end{abstract}

Quantum mechanical entangled configurations of particles that do not satisfy Bell's inequalities, or equivalently, do not have a joint probability distribution, are familiar in the foundational literature of quantum mechanics. Nonexistence of a joint probability measure for the correlations predicted by quantum mechanics is itself equivalent to the nonexistence of local hidden variables that account for the correlations (for a proof of this equivalence, see Suppes and Zanotti, 1981).

From a philosophical standpoint it is natural to ask what sort of concept can be used to provide a "joint" analysis of such quantum correlations. In other areas of application of probability, similar but different problems arise. A typical example is the introduction of upper and lower probabilities in the theory of belief. A person may feel uncomfortable assigning a precise probability to the occurrence of rain tomorrow, but feel comfortable saying the probability should be greater than $1 / 2$ and less than $7 / 8$. Rather extensive statistical developments have occurred for this framework. A thorough treatment can be found in Walley (1991) and an earlier measurement-oriented development in Suppes (1974). It is important to note that this focus on beliefs, or related Bayesian ideas, is not concerned, as we are here, with the nonexistence of joint probability distributions. Yet earlier work with no relation to quantum mechanics, but focused on conditions for existence has been published by many people. For some of our own work on this topic, see Suppes and Zanotti (1989).

Still, this earlier work naturally suggested the question of whether or not upper and lower measures could be used in quantum mechanics, as a generalization of 
probability. To show that an affirmative answer is possible, and, we hope of some philosophical interest, is the general purpose of this paper.

Following Suppes and Zanotti (1991) the initial focus is to construct an upperprobability measure for Bell-type correlations. Such a construction was sketched in the paper just mentioned, but full details are needed here to study the decoherence decay of such systems, our second topic.

Computation of decoherence times is an important feature of decoherence theories. The literature in fact includes specific results on of whether or not most entangled systems of quantum particles have an expected decoherence time that is much too fast for humans or other animals to make any brain computations that are quantum mechanical. (For a skeptical view of this possibility, see Suppes and de Barros, 2007.)

The question of special interest here is whether a computation of decoherence decay of the upper probability measure we construct gives a good approximation of the decay time obtained from direct quantum mechanical calculations of the decoherence decay of the "too active" quantum correlations.

For later use, we give here the definition of upper probability.

Definition 1. Let $\Omega$ be a nonempty set, F a Boolean algebra on $\Omega$, and $P^{*}$ a real-valued $\mathrm{f}$ unction on $\mathrm{F}$. Then $\Omega=\left(\Omega, F, P^{*}\right)$ is an upper probability space if and only if for every $A$ and $B$ in $F$

1. $0 \leqslant P^{*}(A) \leq 1$;

2. $P^{*}(\varnothing)=0$ and $P^{*}(\Omega)=1$;

3. If $A \cap B=\varnothing$, then $P^{*}(A \cup B) \leqslant P^{*}(A)+P^{*}(B)$.

Axiom 3 on finite subadditivity could be strengthened to $\sigma$-subadditivity but we are not concerned with that issue here.

\section{Upper probabilities in quantum mechanics}

We use the standard notation familiar in the Bell inequalities which we review very briefly. For definiteness, but not required, we can think of a Bell-type experiment in which we are measuring spin for particle A and for particle B. More generally, we may think of $\mathrm{A}$ and $\mathrm{B}$ as being the location of measuring equipment and we observe individual particles or a flux of particles at each of the sites. Here we will think of individual particles because the analysis is simpler. The measuring apparatus is such that along the axis connecting A and B we have axial symmetry and consequently we can describe the position of the measuring apparatus just by the angle of the apparatus $\mathrm{A}$ or $\mathrm{B}$ in the plane perpendicular to the axis. We use the notation $w_{A}$ and $w_{B}$ for these angles. The basic form of the locality assumption is shown in terms of the following expectation: 


$$
\mathrm{E}\left(\mathrm{M}_{\mathrm{A}} \mid \mathrm{w}_{\mathrm{A}}, \mathrm{w}_{\mathrm{B}}, \lambda\right)=\mathrm{E}\left(\mathrm{M}_{\mathrm{A}} \mid \mathrm{w}_{\mathrm{A}}, \lambda\right)
$$

What this means is the expectation of the measurement $\mathbf{M}_{\mathrm{A}}$, of spin of a particle in the apparatus in position $A$, given the two angles of measurement for apparatus $A$ and $B$ as well as the hidden variable $\lambda$, is equal to the expectation without knowledge of the apparatus angle $w_{\mathrm{B}}$, of $B$. This is a reasonable causal assumption and is a way of saying that what happens at $B$ should have no direct causal influence on what happens at $A$. On the other hand, we have the following theoretical result for spin, well confirmed in principle for the case where the measuring apparatuses are both set at the same angle:

$$
\mathrm{P}\left(\mathrm{M}_{\mathrm{A}}=-1 \mid \mathrm{w}_{\mathrm{A}}=\mathrm{w}_{\mathrm{B}}=\mathrm{x} \& \mathrm{M}_{\mathrm{B}}=1\right)=1
$$

If the angles of the apparatus are set the same, we have a deterministic result in the sense that the observation of an EPR state at $B$ will be the opposite at $A$, and conversely. Here we are letting 1 correspond to spin $1 / 2$ and -1 correspond to spin $-1 / 2$. What Bell showed is that on the assumption there exists a hidden variable, four related inequalities can be derived for settings $A$ and $A^{\prime}$ and $B$ and $B^{\prime}$ for the measuring apparatus. We have reduced the notation here in the following way in writing the inequalities. First, instead of writing $\mathbf{M}_{\mathrm{A}}$, we write simply $\mathbf{A}$, and second, instead of writing $\operatorname{Cov}(\mathbf{A}, \mathbf{B})$ for the covariance, which in this case will be the same as the correlation, of the measurement at $A$ and the measurement at $B$, we write simply AB. With this understanding about the conventions of the notation, we then have as a consequence of the assumption of a hidden variable the following set of inequalities, which in the exact form given here are due to Clauser, Horne, Shimony, and Holt (1969):

$$
\begin{aligned}
& -2 \leqslant \mathbf{A} \mathbf{B}+\mathbf{A} \mathbf{B}^{\prime}+\mathbf{A}^{\prime} \mathbf{B}-\mathbf{A}^{\prime} \mathbf{B}^{\prime} \leqslant 2 \\
& -2 \leqslant \mathbf{A} \mathbf{B}+\mathbf{A} \mathbf{B}^{\prime}-\mathbf{A}^{\prime} \mathbf{B}+\mathbf{A}^{\prime} \mathbf{B}^{\prime} \leqslant 2 \\
& -2 \leqslant \mathbf{A} \mathbf{B}-\mathbf{A} \mathbf{B}^{\prime}+\mathbf{A}^{\prime} \mathbf{B}+\mathbf{A}^{\prime} \mathbf{B}^{\prime} \leqslant 2 \\
& -2 \leqslant-\mathbf{A B}+\mathbf{A} \mathbf{B}^{\prime}+\mathbf{A}^{\prime} \mathbf{B}+\mathbf{A}^{\prime} \mathbf{B}^{\prime} \leqslant 2
\end{aligned}
$$

Quantum mechanics does not satisfy these inequalities in general. To illustrate ideas, we take as a particular case the following:

$$
\mathbf{A B}-\mathbf{A} \mathbf{B}^{\prime}+\mathbf{A}^{\prime} \mathbf{B}+\mathbf{A}^{\prime} \mathbf{B}^{\prime}<-2
$$

We choose

$$
\mathbf{A B}=\mathbf{A}^{\prime} \mathbf{B}^{\prime}=-\cos 30^{\circ}=-\frac{\sqrt{3}}{2}
$$




$$
\begin{aligned}
& \mathbf{A B}^{\prime}=-\cos 60^{\circ}=-\frac{1}{2} \\
& \mathbf{A}^{\prime} \mathbf{B}=-\cos 0^{\circ}=-1
\end{aligned}
$$

So the inequality $(3 \mathrm{c})$ is violated by this example, since from quantum mechanics $\operatorname{Cov}(\mathbf{A B})=-\cos ($ angle $\mathbf{A B})$ and

$$
-\frac{\sqrt{3}}{2}+\frac{1}{2}-1-\frac{\sqrt{3}}{2}<-2 .
$$

First we must compute the probabilities for the pairs with given correlations, using dots for missing arguments. So

$$
\mathrm{P}(\mathbf{A}=1)=p(1 \cdot \cdots)=p(-1 \cdots)=\frac{1}{2}
$$

since $E(A)=0$, and by similar arguments and notation

$$
\mathrm{P}\left(\mathbf{B}^{\prime}=1\right)=p(\cdots 1)=p(\cdots-1)=\frac{1}{2}
$$

For ease of reading we replace " -1 " by " 0 ".

Now the correlation

$$
\mathbf{A B}=-\frac{\sqrt{3}}{2}
$$

so

$$
-\frac{\sqrt{3}}{2}=p(1 \cdot 1 \cdot)+p(0 \cdot 0 \cdot)-p(1 \cdot 0 \cdot)-p(0 \cdot 1 \cdot)
$$

But by symmetry

$$
p(1 \cdot 1 \cdot)=p(0 \cdot 0 \cdot)
$$

and

$$
p(1 \cdot 0 \cdot)=p(0 \cdot 1 \cdot) .
$$

So solving, we obtain

$$
4 p(1 \cdot 1 \cdot)-1=\frac{\sqrt{3}}{2}
$$


and

$$
\begin{aligned}
& p(1 \cdot 1 \cdot)=-\frac{\sqrt{3}}{8}+\frac{1}{4} \\
& p(1 \cdot 0 \cdot)=\frac{\sqrt{3}}{8}+\frac{1}{4} .
\end{aligned}
$$

Similarly for $\mathbf{A}^{\prime} \mathbf{B}^{\prime}=-\frac{\sqrt{3}}{2}$

$$
\begin{gathered}
\mathrm{P}\left(\mathbf{A}=1, \mathbf{B}^{\prime}=1\right)=p(\cdot 1 \cdot 1)=-\frac{\sqrt{3}}{8}+\frac{1}{4} \\
p(\cdot 1 \cdot 0)=\frac{\sqrt{3}}{8}+\frac{1}{4} .
\end{gathered}
$$

Next $\mathbf{A B}^{\prime}=1 / 2$, so

$$
\begin{aligned}
4 p(1 \cdots 1)-1 & =-\frac{1}{2} \\
p(1 \cdots 1) & =\frac{1}{8} \\
p(1 \cdots 0) & =\frac{3}{8} .
\end{aligned}
$$

Since $\mathbf{A}^{\prime} \mathbf{B}=-1$

$$
\begin{aligned}
& 4 p(\cdot 11 \cdot)-1=-1 \\
& p(\cdot 11 \cdot)=0 \\
& p(\cdot 10 \cdot)=\frac{1}{2} .
\end{aligned}
$$

Since each of the four measurements $\mathbf{A}, \mathbf{A}^{\prime}, \mathbf{B}$, and $\mathbf{B}^{\prime}$ has value \pm 1 , there are 16 atoms, i.e., atomic events, in our upper probability space $\Omega$. There are not simple elementary probability arguments of the kind we have just been following, to compute the upper probability of these atoms. The reason is simple; the main 
probabilistic law that must be preserved is the subadditivity of upper probabilities, expressed as Axiom 3 of Definition 1. This axiom is, of course, weaker than the standard additivity axiom. If we held onto the standard additivity and use the methods just used for computing probabilities of correlations, we would have atoms with negative probabilities, the sort of thing that happens in quantum mechanics when using the Wigner distribution for position and momentum of a single particle (for details on this, see Suppes, 1961).

So, to make what could easily be a longer story short, here are the upper probabilities for the axioms. Since $\mathrm{E}(\mathbf{A})=\mathrm{E}\left(\mathbf{A}^{\prime}\right)=\mathrm{E}(\mathbf{B})=\mathrm{E}\left(\mathbf{B}^{\prime}\right)=0$, by symmetry we need find only 8 . Here is the list.

$$
\begin{aligned}
& p^{*}\left(\begin{array}{llll}
1 & 1 & 1 & 1
\end{array}\right)=p^{*}\left(\begin{array}{llll}
0 & 0 & 0 & 0
\end{array}\right)=0 \\
& p^{*}\left(\begin{array}{llll}
1 & 1 & 1 & 0
\end{array}\right)=p^{*}\left(\begin{array}{llll}
0 & 0 & 0 & 1
\end{array}\right)=\frac{1}{16} \\
& p^{*}\left(\begin{array}{llll}
1 & 1 & 0 & 1
\end{array}\right)=p^{*}\left(\begin{array}{llll}
0 & 0 & 1 & 0
\end{array}\right)=\frac{1}{8} \\
& p^{*}\left(\begin{array}{llll}
1 & 1 & 0 & 0
\end{array}\right)=p^{*}\left(\begin{array}{llll}
0 & 0 & 1 & 1
\end{array}\right)=\frac{1}{8}+\frac{\sqrt{3}}{8} \\
& p^{*}\left(\begin{array}{llll}
1 & 0 & 1 & 0
\end{array}\right)=p^{*}\left(\begin{array}{llll}
0 & 1 & 0 & 1
\end{array}\right)=\frac{1}{8}-\frac{\sqrt{3}}{8} \\
& p^{*(}\left(\begin{array}{llll}
1 & 0 & 1 & 1
\end{array}\right)=p^{*}\left(\begin{array}{llll}
0 & 1 & 0 & 0
\end{array}\right)=\frac{1}{8} \\
& p^{*}\left(\begin{array}{llll}
1 & 0 & 0 & 0
\end{array}\right)=p^{*}\left(\begin{array}{llll}
0 & 1 & 1 & 1
\end{array}\right)=\frac{1}{16} \\
& p^{*(}\left(\begin{array}{llll}
1 & 0 & 0 & 1
\end{array}\right)=p^{*}\left(\begin{array}{llll}
0 & 1 & 1 & 0
\end{array}\right)=0
\end{aligned}
$$

Note that the upper probabilities are non-negative and not greater than 1 . What makes them as a whole upper probabilities, not standard probabilities, is that the sum of the 16 is greater than 1:

$$
\sum_{i, j, k, l=0}^{1} p^{*}(i, j, k, l)=1+\frac{3}{8}
$$


We now verify that each of the correlation probabilities computed earlier satisfy the subadditivity for the four of the 16 atoms that define it as an event. To

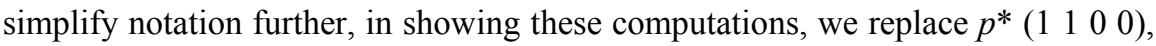
e.g., by 1100 . So all the following inequalities are really about upper probabilities, but " $p$ *" has been deleted.

$$
\begin{aligned}
\text { AB: } p(1 \cdot 0 \cdot)=\frac{1}{4}+\frac{\sqrt{3}}{8} & \leq 1101+1100+1001+1000 \\
& \leq \frac{1}{8}+\left(\frac{1}{8}+\frac{\sqrt{3}}{8}\right)+\frac{1}{16}+\frac{1}{16} \\
& \leq \frac{3}{8}+\frac{\sqrt{3}}{8} \\
p(1 \cdot 1 \cdot)=-\frac{1}{4}-\frac{\sqrt{3}}{8} \leq & 111+1110+1011+1010 \\
\leq & 0+\frac{1}{16}+\frac{1}{8}+\left(\frac{1}{8}-\frac{\sqrt{3}}{8}\right) \\
\leq & \frac{5}{16}-\frac{\sqrt{3}}{8} .
\end{aligned}
$$

$\mathbf{A B}^{\prime}: \quad p(1 \cdots 0)=\frac{3}{8} \leq 1110+1100+1010+1000$

$$
\begin{aligned}
& \leq \frac{1}{16}+\left(\frac{1}{8}+\frac{\sqrt{3}}{8}\right)+\frac{1}{8}-\left(\frac{\sqrt{3}}{8}+\frac{1}{16}\right) \\
& \leq \frac{3}{8} \\
p(1 \cdots 1)=\frac{1}{8} & \leq 1111+1101+1011+1001 \\
& \leq 0+\frac{1}{8}+\frac{1}{8}+\frac{1}{16} \\
& \leq \frac{5}{16} .
\end{aligned}
$$


$\mathbf{A}^{\prime} \mathbf{B}: \quad p(\cdot 10 \cdot)=\frac{1}{2} \leq 1101+1100+0101+0100$

$$
\begin{aligned}
& \leq \frac{1}{8}+\left(\frac{1}{8}+\frac{\sqrt{3}}{8}\right)+\left(\frac{1}{8}-\frac{\sqrt{3}}{8}\right)+\frac{1}{8} \\
& \leq \frac{1}{2} \\
& p(\cdot 11 \cdot)=0 \leq 1111+1110+0111+0110 \\
& \leq 0+\frac{1}{16}+\frac{1}{16}+\frac{1}{16} \\
& \leq \frac{3}{16} \text {. } \\
& \mathbf{A}^{\prime} \mathbf{B}^{\prime}: \quad p(\cdot 1 \cdot 0)=\frac{1}{4}+\frac{\sqrt{3}}{8} \leq 1110+1100+0110+0100 \\
& \leq \frac{1}{16}+\left(\frac{1}{8}+\frac{\sqrt{3}}{8}\right)+\frac{1}{16}+\frac{1}{8} \\
& \leq \frac{3}{8}+\frac{\sqrt{3}}{8} \\
& p(\cdot 1 \cdot 1)=-\frac{1}{4}-\frac{\sqrt{3}}{8} \leq 1111+1101+0111+0101 \\
& \leq 0+\frac{1}{8}+\frac{1}{16}+\left(\frac{1}{8}-\frac{\sqrt{3}}{8}\right) \\
& \leq \frac{5}{16}-\frac{\sqrt{3}}{8} \text {. }
\end{aligned}
$$

\section{The decay of the EPR state and the existence of a joint} distribution

We calculate the time evolution of the EPR state 


$$
\mid \mathrm{EPR}>=\frac{1}{\sqrt{2}}(|01>-| 10>)
$$

under the influence of decoherence. The decaying state will not stay pure, but become mixed in the course of time. We therefore calculate the corresponding initial density operator and $\mathrm{P}(0):=|\mathrm{EPR}><\mathrm{EPR}|$ obtain

$$
P(0)=\frac{1}{2}(|01><01|+|10><10|)-\frac{1}{2}(|01><10|+|10><01|)
$$

There are many different ways to model the influence of decoherence on a quantum system described by a quantum state (Schlosshauer 2007). Here we focus on the master equation approach that is popular in quantum optics. According to this approach, a quantum state couples to an environment ("heat bath"), which is modeled as an infinite collection of harmonic oscillators. This way of modeling decoherence takes into account that a quantum system can never be shielded from its environment (Zeh 1973). Note that due to the coupling of the quantum system to the environment, the entanglement of the quantum system in question diffuses into the environment and the reduced state of the system becomes less and less entangled. This reduced state of the quantum system can be obtained by a procedure called "tracing out" the environment variables. This procedure can be justified by noting that nothing is known about the environment and so it is appropriate to take a statistical average. Finally, one obtains a master equation for the reduced state $P$ of our 2-atom system,

$$
\frac{\partial}{\partial t} P(t)=-\frac{k}{2} \sum_{i=1}^{2}\left[\sigma_{+}^{(i)} \sigma_{-}^{(i)} P(t)+P(t) \sigma_{+}^{(i)} \sigma_{-}^{(i)}-2 \sigma_{-}^{(i)} P(t) \sigma_{+}^{(i)}\right]=: L P(t),(6)
$$

with the damping constant $k . \sigma_{ \pm}^{(i)}$ are the raising and lowering operator acting on atom $i$. These operators can be expressed in terms of the Pauli matrices $\sigma_{1}$ and $\sigma_{2}:$

$$
\sigma_{ \pm}=\frac{1}{2}\left(\sigma_{1} \pm \mathrm{i} \sigma_{2}\right)
$$

with 


$$
\sigma_{1}=\left(\begin{array}{ll}
0 & 1 \\
1 & 0
\end{array}\right) \quad, \quad \sigma_{2}=\left(\begin{array}{cc}
0 & -\mathrm{i} \\
\mathrm{i} & 0
\end{array}\right)
$$

Eq.(6) can be formally solved:

$$
P(t)=\mathrm{e}^{L t} P(0)
$$

Using eqs. (5) and (6) and after some algebra (see Hartmann, 2009), we obtain the time evolution of the EPR state,

$$
P(\tau)=e^{-\tau} P(0)+\left(1-e^{-\tau}\right)|00><00|
$$

with the normalized time parameter $\tau=k t$.

We see that the quantum system under consideration asymptotically reaches the ground state $|00><00|$.

Let us now study the correlation that the decaying quantum state exhibits. In order to connect to the discussion in Section 1, we focus on the following four observables:

$$
\begin{aligned}
& \mathbf{A}=\sigma_{1}^{(1)} \\
& \mathbf{A}^{\prime}=\sigma_{1}^{(1)} \cos (\alpha)+\sigma_{2}^{(1)} \sin (\alpha) \\
& \mathbf{B}=\sigma_{1}^{(2)} \cos (\alpha)+\sigma_{2}^{(2)} \sin (\alpha) \\
& \mathbf{B}^{\prime}=\sigma_{1}^{(2)} \cos (\beta)+\sigma_{2}^{(2)} \sin (\beta)
\end{aligned}
$$

Note that $\mathbf{A}$ and $\mathbf{A}^{\prime}$ act only on particle 1 and $\mathbf{B}$ and $\mathbf{B}^{\prime}$ act only on particle 2. Clearly, the expectation values of $\mathbf{A}, \mathbf{A}^{\prime}, \mathbf{B}$ and $\mathbf{B}^{\prime}$ in $P(t)$ all vanish for all times $\tau$ :

$$
\langle\mathbf{A}\rangle=\left\langle\mathbf{A}^{\prime}\right\rangle=\langle\mathbf{B}\rangle=\left\langle\mathbf{B}^{\prime}\right\rangle=0
$$

However, the two-particle correlations $<\mathbf{A} \mathbf{B}>,<\mathbf{A} \mathbf{B}^{\prime}>,<\mathbf{A}^{\prime} \mathbf{B}>$ and $\left\langle\mathbf{A}^{\prime} \mathbf{B}^{\prime}>\right.$ do not vanish. We calculate the expectation values of these operators for the state $\mathrm{P}(\tau)$. 


$$
\begin{aligned}
& <\mathbf{A B}>=-\mathrm{e}^{-\tau} \cos (\alpha) \\
& <\mathbf{A} \mathbf{B}^{\prime}>=-\mathrm{e}^{-\tau} \cos (\beta) \\
& <\mathbf{A}^{\prime} \mathbf{B}>=-\mathrm{e}^{-\tau} \\
& <\mathbf{A}^{\prime} \mathbf{B}^{\prime}>=-\mathrm{e}^{-\tau} \cos (\alpha-\beta)
\end{aligned}
$$

We expect that these correlations can be derived from a joint probability distribution for sufficiently large $\tau$, i.e. when the state is sufficiently decayed. But when precisely is a description of the correlations in terms of a joint probability distribution possible? This question is addressed by the Clauser-Horne-Shimony-Holt inequalities (see eqs. (3)). To be more specific, let $\alpha=30^{\circ}$ and $\beta=60^{\circ}$, the example introduced in Section 1. It turns out that inequalities (3a), (3b) and (3d) are always satisfied. However, inequality (3c) leads to

$$
\mathrm{e}^{-\tau} \leq \frac{4}{2 \sqrt{3}+1}
$$

or $\tau>.1$.

We see that a "classical" description of the correlations is possible already after a very short period of time (in units of $k^{-1}$ ).

Instead of the calculation of (9) for the decay of the quantum mechanical theoretical correlations, we now compute the upper-probability correlations from the upper-probability values of the 16 atoms. We will label these correlations

$<\mathbf{A} \mathbf{B}>*$ (superscript for upper).

So, here are the calculations of the four upper correlations.

$$
\begin{aligned}
& <\mathbf{A ~ B}>*: p^{*}\left(\begin{array}{lll}
1 & \cdot & 1
\end{array}\right)=p^{*}\left(\begin{array}{llll}
1 & 1 & 1 & 1
\end{array}\right)+p^{*}\left(\begin{array}{llll}
1 & 1 & 1 & 0
\end{array}\right)+p^{*}\left(\begin{array}{llll}
1 & 0 & 1 & 1
\end{array}\right)+p^{*}\left(\begin{array}{llll}
1 & 0 & 1 & 0
\end{array}\right) \\
& =0+\frac{1}{16}+\frac{1}{8}+\frac{1}{8}-\frac{\sqrt{3}}{8} \\
& =\frac{5}{16}-\frac{\sqrt{3}}{8} \\
& p^{*}\left(\begin{array}{lll}
1 & \cdot & 0
\end{array}\right)=p^{*}\left(\begin{array}{llll}
1 & 1 & 0 & 1
\end{array}\right)+p^{*}\left(\begin{array}{llll}
1 & 1 & 0 & 0
\end{array}\right)+p^{*}\left(\begin{array}{llll}
1 & 0 & 0 & 1
\end{array}\right)+p^{*}\left(\begin{array}{llll}
1 & 0 & 0 & 0
\end{array}\right) \\
& =\frac{1}{8}+\frac{1}{8}+\frac{\sqrt{3}}{8}+0+\frac{1}{16}
\end{aligned}
$$




$$
\begin{aligned}
& =\frac{5}{16}+\frac{\sqrt{3}}{8} \\
\text { so }<\mathbf{A} \mathbf{B}>* & =2\left[\frac{5}{16}-\frac{\sqrt{3}}{8}-\left(\frac{5}{16}+\frac{\sqrt{3}}{8}\right)\right] \\
& =-\frac{\sqrt{3}}{2} .
\end{aligned}
$$

$<\mathbf{A} \mathbf{B}^{\prime}>^{*}: p^{*}\left(\begin{array}{lll}1 & \cdots & 1\end{array}\right)=p^{*}\left(\begin{array}{llll}1 & 1 & 1 & 1\end{array}\right)+p^{*}\left(\begin{array}{llll}1 & 1 & 0 & 1\end{array}\right)+p^{*}\left(\begin{array}{llll}1 & 0 & 1 & 1\end{array}\right)+p^{*}\left(\begin{array}{llll}1 & 0 & 0 & 1\end{array}\right)$

$$
\begin{aligned}
& =0+\frac{1}{8}+\frac{1}{8}+0 \\
& =\frac{1}{4} .
\end{aligned}
$$

$p^{*}\left(\begin{array}{lll}1 & \cdots & 0\end{array}\right)=p^{*}\left(\begin{array}{llll}1 & 1 & 1 & 0\end{array}\right)+p^{*}\left(\begin{array}{llll}1 & 1 & 0 & 0\end{array}\right)+p^{*}\left(\begin{array}{llll}1 & 0 & 1 & 0\end{array}\right)+p^{*}\left(\begin{array}{llll}1 & 0 & 0 & 0\end{array}\right)$

$$
\begin{aligned}
& =\frac{1}{16}+\frac{1}{8}+\frac{\sqrt{3}}{8}+\frac{1}{8}-\frac{\sqrt{3}}{8}+\frac{1}{16} \\
& =\frac{3}{8} \\
& \text { so }<\mathbf{A} \mathbf{B}^{\prime}>*=2\left[\frac{1}{4}-\frac{3}{8}\right] \\
& =-\frac{1}{4} \text {. } \\
& <\mathbf{A}^{\prime} \mathbf{B}>^{*}: p^{*}\left(\begin{array}{lll}
\cdot & 1 & 1
\end{array}\right)=p^{*}\left(\begin{array}{llll}
1 & 1 & 1 & 1
\end{array}\right)+p^{*}\left(\begin{array}{llll}
1 & 1 & 1 & 0
\end{array}\right)+p^{*}\left(\begin{array}{llll}
0 & 1 & 1 & 1
\end{array}\right)+p^{*}\left(\begin{array}{llll}
0 & 1 & 1 & 0
\end{array}\right) \\
& =0+\frac{1}{16}+\frac{1}{16}+0
\end{aligned}
$$




$$
=\frac{1}{8} \text {. }
$$

$p^{*}\left(\cdot \begin{array}{lll}1 & 0\end{array}\right)=p^{*}\left(\begin{array}{llll}1 & 1 & 0 & 1\end{array}\right)+p^{*}\left(\begin{array}{llll}1 & 1 & 0 & 0\end{array}\right)+p^{*}\left(\begin{array}{llll}0 & 1 & 0 & 1\end{array}\right)+p^{*}\left(\begin{array}{llll}0 & 1 & 0 & 0\end{array}\right)$

$$
\begin{aligned}
& =\frac{1}{8}+\frac{1}{8}+\frac{\sqrt{3}}{8}+\frac{1}{8}-\frac{\sqrt{3}}{8}+\frac{1}{8} \\
& =\frac{1}{2}
\end{aligned}
$$

so $\left\langle\mathbf{A}^{\prime} \mathbf{B}>*=2\left[\frac{1}{8}-\frac{1}{2}\right]\right.$

$$
=-\frac{3}{4} \text {. }
$$

$<\mathbf{A}^{\prime} \mathbf{B}^{\prime}>^{*}: p^{*}\left(\cdot \begin{array}{ll}\cdot 1 \cdot 1\end{array}\right)=p^{*}\left(\begin{array}{llll}1 & 1 & 1 & 1\end{array}\right)+p^{*}\left(\begin{array}{llll}1 & 1 & 0 & 1\end{array}\right)+p^{*}\left(\begin{array}{llll}0 & 1 & 1 & 1\end{array}\right)+p^{*}\left(\begin{array}{llll}0 & 1 & 0 & 1\end{array}\right)$

$$
\begin{aligned}
& =0+\frac{1}{8}+\frac{1}{16}+\frac{1}{8}-\frac{\sqrt{3}}{8} \\
& =\frac{5}{16}-\frac{\sqrt{3}}{8}
\end{aligned}
$$

$p^{*}(\cdot 1 \cdot 0)=p^{*}\left(\begin{array}{llll}1 & 1 & 1 & 0\end{array}\right)+p^{*}\left(\begin{array}{llll}1 & 1 & 0 & 0\end{array}\right)+p^{*}\left(\begin{array}{llll}0 & 1 & 1 & 0\end{array}\right)+p^{*}\left(\begin{array}{llll}0 & 1 & 0 & 0\end{array}\right)$

$$
\begin{aligned}
& =\frac{1}{16}+\frac{1}{8}+\frac{\sqrt{3}}{8}+0+\frac{1}{8} \\
& =\frac{5}{16}+\frac{\sqrt{3}}{8} \\
\text { so }\left\langle\mathbf{A}^{\prime} \mathbf{B}^{\prime}>*\right. & =2\left[\frac{5}{16}-\frac{\sqrt{3}}{8}-\left(\frac{5}{16}+\frac{\sqrt{3}}{8}\right)\right]
\end{aligned}
$$




$$
=-\frac{\sqrt{3}}{2}
$$

Putting these upper correlations into inequality (3c), we get

$$
-\frac{\sqrt{3}}{2}+\frac{1}{4}-\frac{3}{4}-\frac{\sqrt{3}}{2}=-\frac{1}{2}-\sqrt{3}<-2 .
$$

Applying then the same decay rate $e^{-\tau}$, we have

$$
\begin{aligned}
& \left(-\frac{1}{2}-\sqrt{3}\right) e^{-\tau} \leq-2, \\
& \text { so } e^{-\tau} \leq \frac{4}{2 \sqrt{3}+1} .
\end{aligned}
$$

exactly the same inequality for the decay time as was obtained earlier for the quantum mechanical computation. Yet the two methods are not identical. It is clear that the two proper joint probability distributions at the time $e^{-\tau}=\frac{4}{1+2 \sqrt{3}}$ are close but not exactly the same.

\section{References}

Clauser, J.F., M. A. Horne, A. Shimony, and R. A. Holt. 1969. Proposed experiment to test local hidden-variable theories. Physics Review Letter, 23, pp. 880-884.

Hartmann, S. 2009. Quantum optical master equations for $Z$ atoms: The use of superoperator methods. Unpublished manuscript. Tilburg University.

Schlosshauer, M. 2007. Decoherence and the quantum-to-classical transition. Springer: Berlin, Heidelberg, New York.

Suppes, P. 1961. Probabilistic concepts in quantum mechanics. Philosophy of Science, 28, 4, pp. 378-389.

Suppes, P. 1974. The measurement of belief. Journal of the Royal Statistical Society (Series B), 36, pp. 160-191.

Suppes, P. and de Barros, J. A. 2007. Quantum mechanics and the brain. Quantum Interaction: Papers from the AAAI Spring Symposium, Technical Report SS-07-08. Menlo Park, CA: AAAI Press, pp. 75-82.

Suppes, P., and Zanotti, M. 1981. When are probabilistic explanations possible? Synthese, 48, pp. 191-199. 
Suppes, P., and Zanotti, M. 1989. Conditions on upper and lower probabilities to imply probabilities. Erkenntnis, 31, pp. 323-345.

Suppes, P., and Zanotti, M. 1991. Existence of hidden variables having only upper probabilities. Foundations of Physics, 21, pp. 1479-1499.

Walley, P. 1991. Statistical reasoning with imprecise probabilities. London, New York: Chapman and Hall.

Zeh, H. D. 1973. Toward a quantum theory of observation. Foundations of Physics, 3, pp. 109116. 